
ergonomics for supermicrosurgery in lymphedema

\author{
Patrick A. Will ${ }^{1,2}$, Christoph Hirche ${ }^{1,2,3}$, Juan Enrique Berner ${ }^{4,5}$, Ulrich Kneser ${ }^{1,2}$, \\ Emre Gazyakan ${ }^{1,2}$ \\ ${ }^{1}$ Department of Hand, Plastic, and Reconstructive Surgery, Microsurgery, Burn Centre, BG-Trauma Hospital Ludwigshafen, Ludwigshafen am \\ Rhein; ${ }^{2}$ Department of Plastic Surgery, Medical Faculty, University of Heidelberg, Heidelberg; ${ }^{3}$ Department of Plastic, Hand, and \\ Reconstructive Microsurgery, Hand Trauma and Replantation Center, BG Unfallklinik Frankfurt am Main, Goethe University Frankfurt, \\ Frankfurt am Main, Germany; ${ }^{4}$ Kellogg College, University of Oxford, Oxford; ${ }^{5}$ Department of Plastic Surgery, Royal Victoria Infirmary, \\ Newcastle upon Tyne, UK
}

\begin{abstract}
The conventional approach of looking down a microscope to perform microsurgical procedures is associated with occupational injuries, anti-ergonomic postures, and increased tremor and fatigue, all of which predispose microsurgeons to early retirement. Recently, three-dimensional (3D) visualization of real-time microscope magnification has been developed as an alternative. Despite its commercial availability, no supermicrosurgical procedures have been reported using this technology to date. Lymphovenous anastomoses (LVAs) often require suturing vessels with diameters of $0.2-0.8 \mathrm{~mm}$, thus representing the ultimate microsurgical challenge. After performing the first documented LVA procedure using 3D-augmented visualization in our unit and gaining experience with this technique, we conducted an anonymized in-house survey among microsurgeons who had used this approach. The participants considered that 3D visualization for supermicrosurgery was equivalent in terms of handling, optical detail, depth resolution, and safety to conventional binocular magnification. This survey revealed that team communication, resident education, and ergonomics were superior using 3D digital hybrid visualization. Postoperative muscle fatigue, tremor, and pain were also reduced. The major drawbacks of the 3D visualization microscopic systems are the associated costs, required space, and difficulty of visualizing the lymphatic contrast used.
\end{abstract}

Keywords Lymphedema / Microsurgery / Ergonomics / Digital hybrid visualization / Imaging, three-dimensional

\author{
Correspondence: \\ Patrick A. Will \\ Department of Hand, Plastic, and \\ Reconstructive Surgery, Microsurgery, \\ Burn Centre, BG-Trauma Hospital \\ Ludwigshafen, Ludwig Guttmann Str. \\ 13, Ludwigshafen am Rhein 67071, \\ Germany \\ Tel: +49-621-68102944 \\ Fax: +49-621-68102617 \\ E-mail: \\ p.will-marks@bgu-ludwigshafen.de
}

\section{Emre Gazyakan}

Department of Hand, Plastic, and

Reconstructive Surgery, Microsurgery, Burn Centre, BG-Trauma Hospital Ludwigshafen, Ludwig Guttmann Str. 13, Ludwigshafen am Rhein 67071, Germany

Tel: +49-621-68102944

Fax: +49-621-68102617

E-mail:

emre.gazyakan@bgu-ludwigshafen.de

Received: October 12, 2020 - Revised: February 17, 2021 • Accepted: March 26, 2021

pISSN: 2234-6163 • elSSN: 2234-6171 • https://doi.org/10.5999/aps.2020.01949 • Arch Plast Surg 2021;48:427-432

\section{INTRODUCTION}

Over the last three decades, traditional surgical practice has adopted the use of innovative technologies that have been demon- strated to achieve better patient outcomes with less invasive interventions. While endoscopic and robotic procedures have been embraced by other surgical specialties, the delivery of microsurgery has not changed much over the last 40 years. Con- 
ventional microsurgery still involves a surgeon and an assistant performing an exacting procedure while looking down the binoculars of a microscope.

This set-up has shown to be associated with occupational injuries due to anti-ergonomic postures and communicative barriers. Studies have revealed that the mean neck flexion during conventional microsurgery is on average more than $36^{\circ}$, which is well beyond the safe limit of $15^{\circ}$, making cervical pain and lesions common [1]. During microsurgery, the equipment constraints, body position, and nature of the procedure result in rigid movements ( 0.3 movements per minute) [2]. This anti-ergonomic posture has been associated with significant muscle fatigue in intraoperative electromyographic studies and increases in hand tremor of about $7 \%$ per surgical hour [2]. Consequently, the prevalence of symptomatic musculoskeletal injuries has been reported to be $77 \%$ in plastic surgeons, while in the general population the prevalence ranges between $0.1 \%$ and $0.4 \%[2,3]$. According to the literature, the anti-ergonomic microsurgical position results not only in occupational injuries, but also in extended sick leave, decreased productivity, and $9 \%$ of early retire- ments $[2,4]$.

Even though robotic-assisted microsurgery has been reported in the literature, its widespread use has not been achieved. This is in part related to unacceptably high rates of complications in preliminary reports [5], which also showed that this technique was excessively time-consuming without achieving better outcomes [6]. Tools were considered not versatile enough to manipulate tissues with different physical properties, and the lack of haptic feedback was thought to account for the excessive rate of technical errors [7]. Furthermore, the use of robotic-assisted microsurgery requires an initial investment of at least 2 million dollars, followed by the cost of consumables ranging between $\$ 1,800$ and $\$ 4,600$ for each surgery [7].

Three-dimensional (3D) video displays and digital augmented reality glasses offer an interesting alternative for microsurgeons. Pilot studies using this technology revealed no differences in perceived effort or qualitative outcomes between $3 \mathrm{D}$ video displays and classic microsurgical training [4], while significantly improving ergonomics and postural shifts [4]. However, the use of $3 \mathrm{D}$ digital hybrid visualization for performing super-micro-

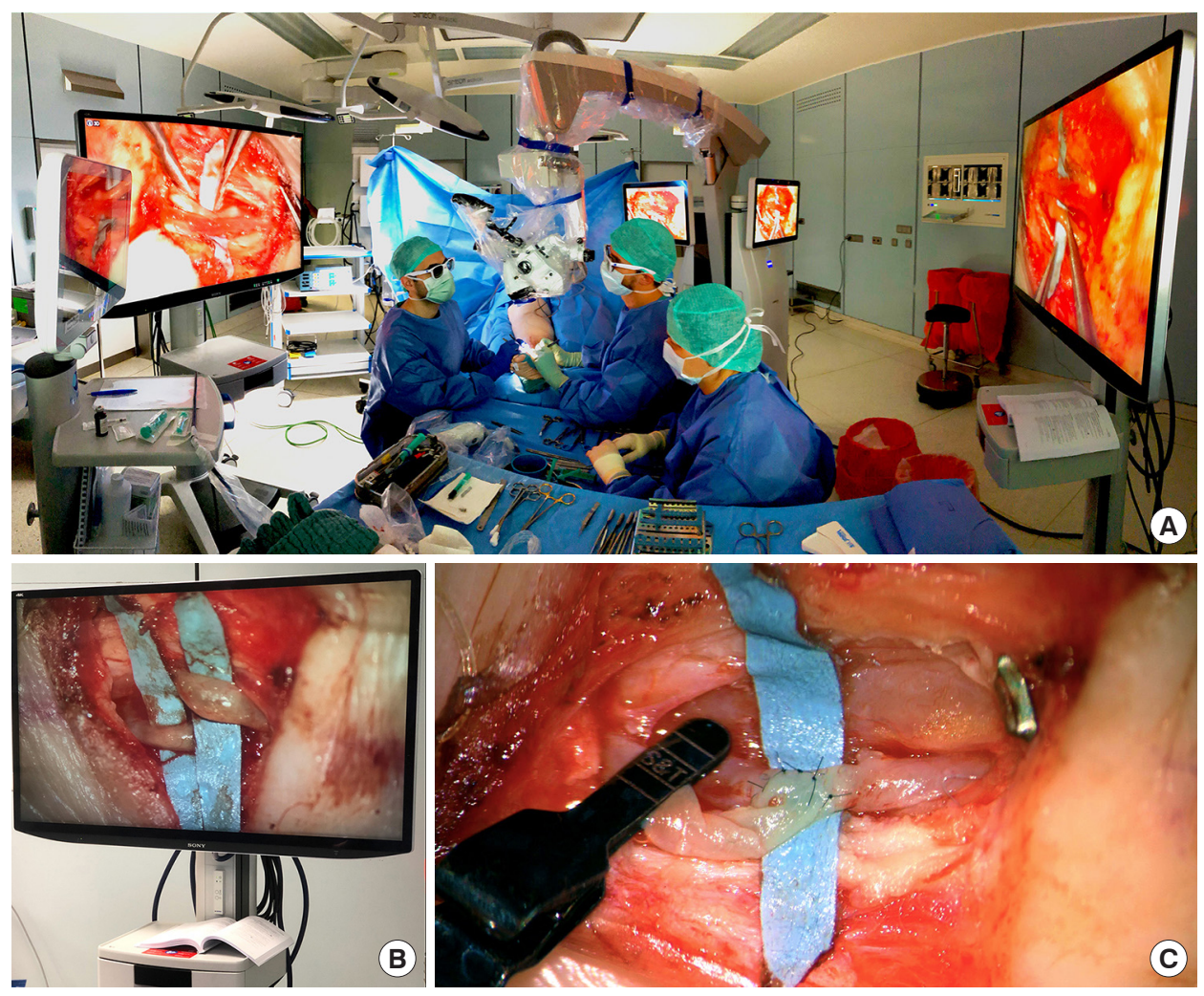

Fig. 1. Operational setting for three-dimensional (3D) digital hybrid visualization supermicrosurgery. (A) The overall setting for 3D digitally augmented lymphovenous anastomosis is shown. The main surgeon and assistant performed supermicrosurgery visualizing opposed monitors, using special glasses provided by the manufacturer. Additional monitors permitted real-time 3D visualization for students, residents, and rest of the surgical team. (B) An example of intraoperative 3D visualization of two lymphatic collectors is presented. This photograph fails to reflect the high-definition of real-life monitors, as glasses are required for best visualization. (C) An example of a lymphovenous anastomosis resulting from 3D-augmented supermicrosurgery is shown. 
surgical procedures has not been previously reported. The aim of this study was to present the authors' early experiences using this technology for performing lymphovenous anastomoses (LVAs).

\section{IDEA}

Hybrid visualization microscopy involves the combined use of optics and digital displays. The surgical microscope is positioned over the patient in a traditional fashion. A magnified image of the operative field is projected on large high-resolution screens, which in combination with wearing the manufacturer's glasses, can provide an immersive 3D experience. The surgeon can still rely on looking down the microscope binoculars if there are any problems.

This set-up allows a comfortable ergonomic posture, providing the surgeon with more freedom of movement. Furthermore, it also allows better communication with the rest of the team, who can also follow the surgeon's movement on the screens (Fig. 1A).

Even though the authors have had performed a number of free tissue transfers using this technology, until recently, it was considered that supermicrosurgical LVAs were unachievable under $3 \mathrm{D}$ visualization [8]. The first LVA with 3D digital hybrid visualization was executed at our center using the Zeiss Kinevo 900 (Zeiss, Oberkochen, Germany), with the optical image projected digitally to $3,840 \times 2,160$-pixel resolution $3 \mathrm{D}$-supported screens. The automated focus feature of this microscope proved to be very useful in this setting in combination with the built-in color-guided visualization of the flow and patency of lymphatic contrast using enhanced near-infrared light detection. Unfortunately, fluorescence near-infrared detection is only available through the eyepieces in this particular device. The distance between the operators and the displays was approximately $1.5-2$ $\mathrm{m}$. The manufacturer does not provide specific recommendations regarding the distance to the screens, as this value might vary depending on the size of the employed display, the preference of the operators, and the freedom of movement desired by the team (Fig. 1B).

During the following project, informed consent was obtained from all patients, and the ethics committee of the BG Unfallklinik had no observations regarding the experimental setting. Our first case of LVA using 3D visualization was a 68 -yearold woman with bilateral secondary lymphedema of the lower extremities following oncological therapy for cervical carcinoma in 2012, including radical hysterectomy, bilateral inguinal lymphadenectomy, and adjuvant radiotherapy. The secondary lymphoedema was later aggravated by iatrogenic lymphatic de- struction due to body-lifting operations subsequent to post-bariatric surgery weight loss. Her preoperative body mass index was $27.6 \mathrm{~kg} / \mathrm{m}^{2}$ and clinical examination revealed bilateral stage 2 lower leg lymphedema with pitting edema, fibrotic skin changes, and non-reversible edema. Color duplex sonography ruled out venous insufficiency, and indocyanine green (ICG)-based lymphography demonstrated isolated lymphatic vessels at the foot dorsum and both malleoli, whereas the rest of the extremities showed a pattern of coalesced stardust and diffuse dermal backflow.

During surgery, $100 \mu \mathrm{L}$ of a $5-\mathrm{mg} / \mathrm{mL}$ dilution of ICG was injected subcutaneously in each interdigital space and $1 \mathrm{~cm}$ inferolateral to the malleolus medialis and lateralis. An external nearinfrared camera (Fluobeam; Fluoptics, Grenoble, France) was used for preoperative and intraoperative lymphangiography (Fig. 2). The first anastomosis in the dorsum of the foot, of a 0.3-mm lymphatic vessel, was performed using the eyepiece. Subsequent LVAs were then performed in the vicinity, now using the 3D digital visualization mode. The light, detail, and texture were as good as through the eyepieces and the handling proved to be surprisingly uncomplicated. There was no delay in the image transmission to the screens, and the movements of the instruments were perceived as a real-time surgical experience without any latency. Although technically challenging (lymphatics of just $0.2 \mathrm{~mm}$ and veins of $0.4 \mathrm{~mm}$ ), the end-toside anastomoses took 27.3 minutes on average ( \pm 8.6 minutes)

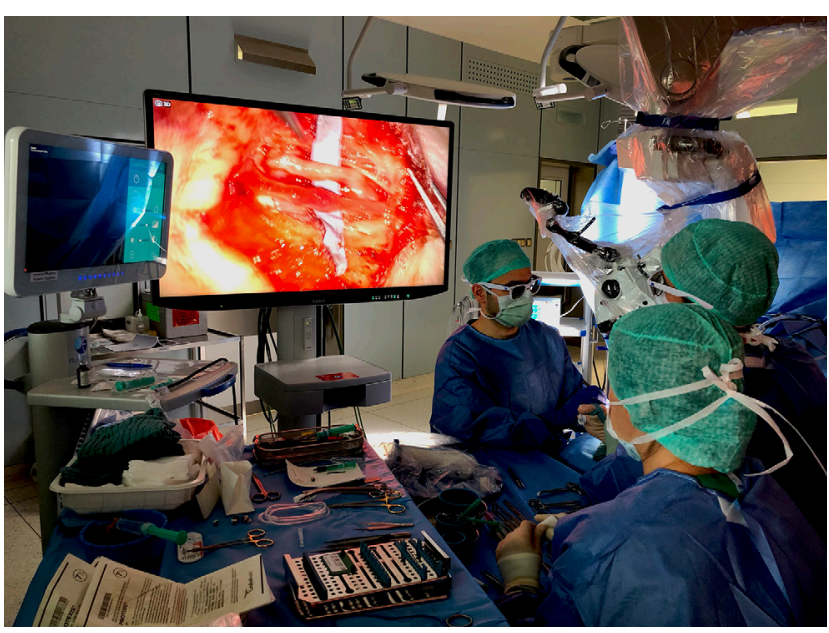

Fig. 2. External near-infrared intraoperative navigation is necessary during three-dimensional (3D) visualized lymphovenous anastomosis surgery. The microscope with 3D digital hybrid visualization presented one significant limitation for supermicrosurgery. It was unable to provide accurate intraoperative lymphatic mapping with subcutaneous application of indocyanine green contrast. During the development of this innovation, an external near-infrared camera was used next to the microscope screens for intraoperative navigation during the lymphovenous anastomoses. 


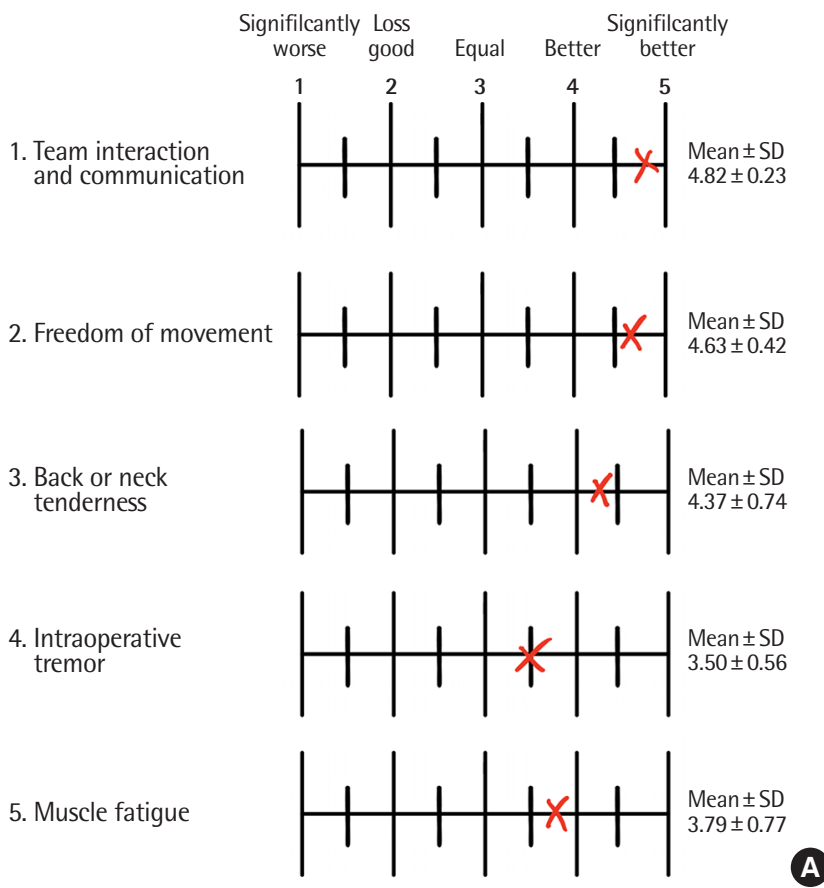

1. Optical detail
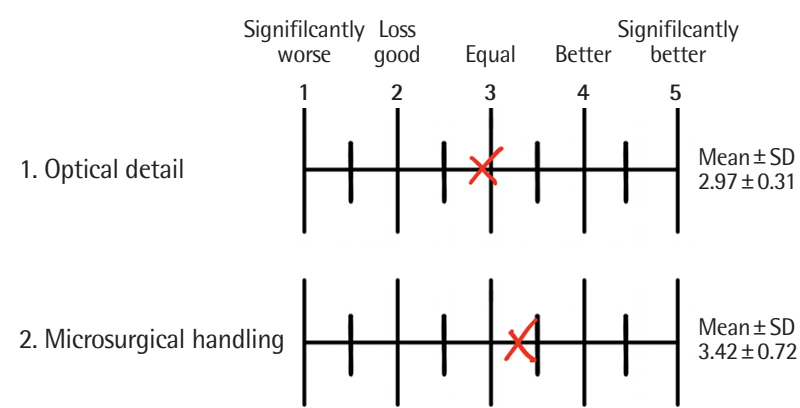
3. Quality of depth and
3D structure visualization

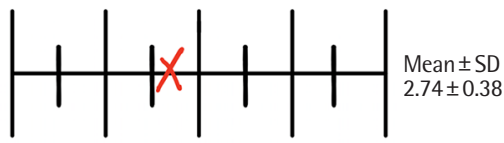

4. Operative comfort

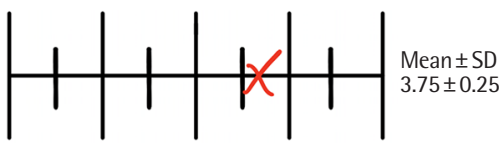

5. Overall satisfaction with the anastomosis

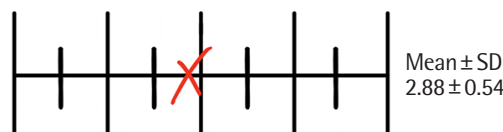

Fig. 3. Results of a survey supporting the use of three-dimensional (3D) digital hybrid visualization in supermicrosurgery. (A) The results of the anonymized survey of 11 supermicrosurgical team members concerning team interaction and ergonomics in 3D and conventional lymphovenous anastomosis (LVA) are shown. The same visualization scale applied during the survey is displayed, as well as the resulting mean and corresponding standard deviation (SD) of the survey. Surgeons considered the use of 3D digital hybrid visualization for LVA surgery to be equal, better, or significantly better than conventional supermicrosurgery in terms of team interaction, communication, and ergonomics. (B) The results of the survey regarding the technical qualities of 3D visualization for supermicrosurgery relative to the conventional method are presented. Depending on the category, the surgical team considered the use of digital hybrid visualization to be slightly better, equal, or marginally less good than the conventional approach.

(Fig. 1C). The overall operative time for five LVAs, removal of lymphoceles, team briefs, breaks, and implementation of this new technology was 8 hours and 42 minutes.

The postoperative LVA protocol was based on soft elastic wraps applied before the patient left the operative theater, followed by manual lymphatic drainage on postoperative day 3 , no compression garments for 3 weeks, and no pneumatic pressure devices for 2 months. Cefuroxime was used for perioperative antibiotic prophylaxis and subcutaneous enoxaparin was prescribed applied until full mobilization. Follow-up examinations took place in the second postoperative week and at postoperative months 3, 6, 12, and 24. We performed four further LVAs using the 3D digital hybrid visualization mode without complications, with similar anastomosis times, and subjectively better communication and less musculoskeletal fatigue (Fig. 3). An average volume reduction of $23 \% \pm 11.7 \%$ was obtained with no complications reported (Fig. 4). The learning curve of consultants and residents turned out to be much faster than expected.

The 11 members of the surgical team involved in performing these procedures completed an anonymized survey (Fig. 3). All surgeons had formal microsurgical training and regular practice at our microsurgical facility. Nearly all of the surveyed surgeons considered that the intraoperative experience performing the dissection and LVA, overall surgical results, optical detail, and microsurgical handling were as good as through binoculars (Fig. 3). All respondents stated that they suffered from pain or muscular fatigue in the arm, fingers, or hand after performing conventional LVA, whereas a major improvement was perceived in terms of less tremor or muscular fatigue during and after 3D LVA surgery (Fig. 3). All respondents conveyed that a key feature of 3D visualization is the increase in musculoskeletal freedom and a general absence of back or neck tenderness (Fig. 3). The surgical team also agreed that performing LVA under 3D digital hybrid visualization significantly improved team communication, resident education, and team interactions compared to conventional supermicrosurgery (Fig. 3).

\section{DISCUSSION}

To our knowledge, this is the first report showing the feasibility of performing supermicrosurgical procedures using 3D digital hybrid visualization microscopy. The microsurgeons involved considered that the technical quality of the employed device provided an experience very similar to the handling and visual- 

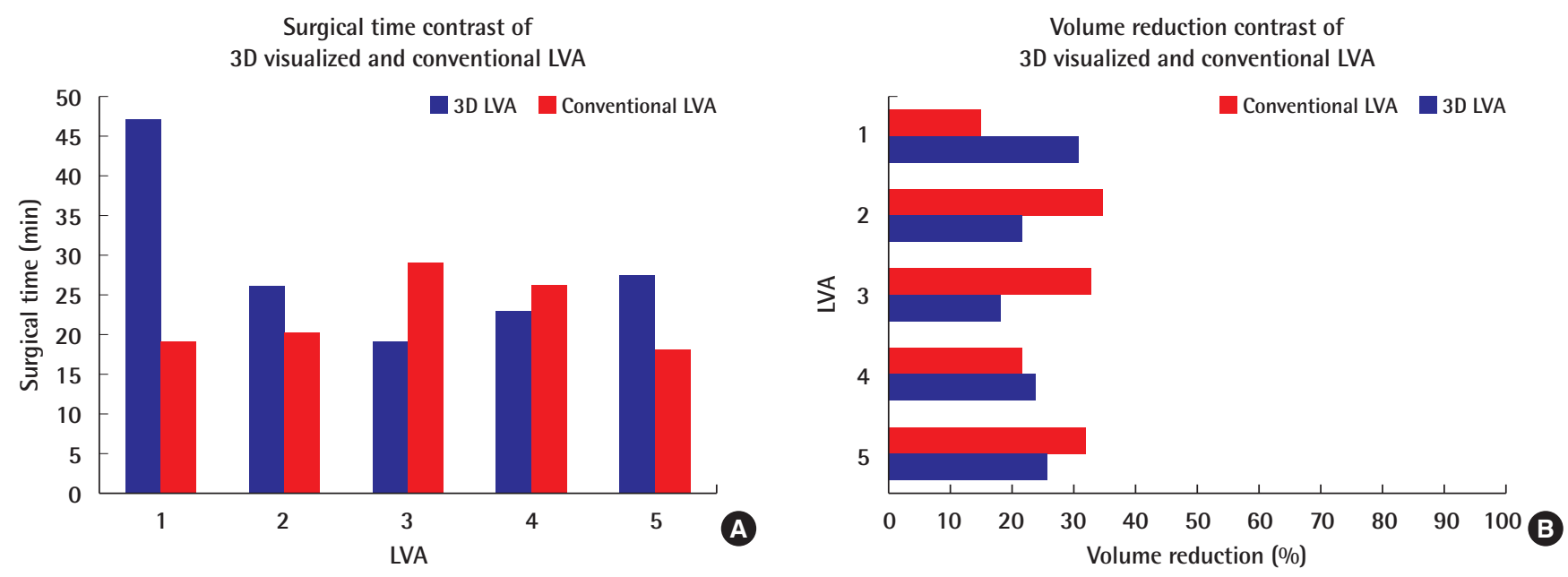

Fig. 4. No major difference in outcomes was found between three-dimensional (3D)-visualized and conventional lymphovenous anastomosis (LVA) surgery. (A) The mean surgical times are shown for the performance of five LVAs using 3D digital hybrid visualization, compared to five LVAs using the conventional approach. The results are representative of the same surgical team and intervention year. With experience, the time required for 3D-visualized LVA was reduced to an extent, with no differences for trained supermicrosurgeons. (B) the mean volume reduction after the first postoperative year is shown for an LVA case performed using 3D digital hybrid visualization and conventional microscopy. On average, a modest volume reduction was achieved with both techniques. Based on the minimal differences and the small group size, no superiority of either approach can be concluded.

ization perceived using conventional means. The subsequent survey revealed that the surgical team viewed this technique as providing significant progress in terms of ergonomic improvement, freedom of movement, and team communication.

Nonetheless, the data must be appraised in relation to the operative time. Each 3D LVA required an average of $27.3 \pm 8.6$ minutes, whereas each conventional supermicrosurgical LVA required on average $22.3 \pm 5.3$ minutes at our unit (Fig. 4 ). The mean overall surgical time of the 3D LVA exceeded the mean conventional LVA surgical time by $23 \%$. This difference could be explained by the necessity of the team to become accustomed to the new technology and might be reduced after more experience. As an example, the very first 3D LVA anastomosis took us 47 minutes, while the following anastomoses required 26 and 19 minutes, respectively (Fig. 4). During this study, we applied the same preoperative and postoperative protocols for the $3 \mathrm{D}$ visualization and conventional LVA procedures to avoid confounding variables.

In sum, 3D digital hybrid visualization effectively enabled secure and uncomplicated LVA, as well as providing comfort and postural freedom, improved ergonomics, and better team interaction. The major drawbacks of current 3D hybrid visualization microscopic systems are the requirement of more space in the operating theater, high costs (about $\$ 500,000$ ), and the fact that contrast visualization is only available through conventional binoculars. The authors believe that future supermicrosurgery might be performed by digital visualization provided by small external 3D optic devices (exoscopes) or head-mounted devices able to zoom in on the operative field. Until then, 3D digital hybrid visualization has passed the proof of principle and might provide the first transitional step to the development of ergonomic robotic-assisted supermicrosurgery.

\section{NOTES}

\section{Conflict of interest}

No potential conflict of interest relevant to this article was reported.

\section{Ethical approval}

The Internal Research Ethics Committee of the BG Unfallklinik Ludwigshafen has confirmed that no ethical approval is required. Written informed consent was obtained.

\section{Patient consent}

The patient provided written informed consent for the publication and the use of her images.

\section{Author contribution}

Conceptualization: PA Will, C Hirche, E Gazyakan. Data curation: JE Berner. Formal analysis: JE Berner. Methodology: PA Will. Project administration: PA Will, U Kneser, E Gazyakan. Visualization: C Hirche. Writing - original draft: PA Will. Writing - review \& editing: C Hirche, JE Berner, U Kneser, E Gazyakan. 


\section{ORCID}

Patrick A. Will https://orcid.org/0000-0003-3430-1795

Christoph Hirche https://orcid.org/0000-0001-6112-8671

Juan Enrique Berner https://orcid.org/0000-0003-2178-5161

Ulrich Kneser https://orcid.org/0000-0002-3402-2860

Emre Gazyakan https://orcid.org/0000-0003-2826-2793

\section{REFERENCES}

1. Yu D, Sackllah M, Woolley C, et al. Quantitative posture analysis of 2D, 3D, and optical microscope visualization methods for microsurgery tasks. Work 2012;41 Suppl 1: 1944-7.

2. Lakhiani C, Fisher SM, Janhofer DE, et al. Ergonomics in microsurgery.J Surg Oncol 2018;118:840-4.

3. Epstein S, Tran BN, Capone AC, et al. Work-related musculoskeletal disorders among plastic surgeons: a systematic re-
view.J Reconstr Microsurg 2018;34:553-62.

4. Yu D, Green C, Kasten SJ, et al. Effect of alternative video displays on postures, perceived effort, and performance during microsurgery skill tasks. Appl Ergon 2016;53 Pt A:2819.

5. Boyd B, Umansky J, Samson M, et al. Robotic harvest of internal mammary vessels in breast reconstruction. J Reconstr Microsurg 2006;22:261-6.

6. Willems JIP, Shin AM, Shin DM, et al. A comparison of robotically assisted microsurgery versus manual microsurgery in challenging situations. Plast Reconstr Surg 2016;137: 1317-24.

7. Tan YPA, Liverneaux P, Wong JKF. Current limitations of surgical robotics in reconstructive plastic microsurgery. Front Surg 2018;5:22.

8. Hong JPJ, Song S, Suh HSP. Supermicrosurgery: principles and applications.J Surg Oncol 2018;118:832-9. 